\title{
Human Pasteurellosis Health Risk for Elderly Persons Living with Companion Animals
}

Sándor Körmöndi, Gabriella Terhes, Zoltán Pál, Endre Varga, Mária Harmati, Kriszina Buzás, Edit Urbán

\section{$\underset{\text { MDUCATION }}{\text { Medscape ACTIVITY }}$}

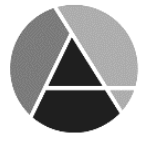

JOINTLY ACCREDITED PROVIDER ${ }^{\text {N }}$ INTERPROFESSIONAL CONTINUING EDUCATION

In support of improving patient care, this activity has been planned and implemented by Medscape, LLC and Emerging Infectious Diseases. Medscape, LLC is jointly accredited by the Accreditation Council for Continuing Medical Education (ACCME), the Accreditation Council for Pharmacy Education (ACPE), and the American Nurses Credentialing Center (ANCC), to provide continuing education for the healthcare team.

Medscape, LLC designates this Journal-based CME activity for a maximum of 1.00 AMA PRA Category 1 Credit(s) ${ }^{\mathrm{TM}}$. Physicians should claim only the credit commensurate with the extent of their participation in the activity.

All other clinicians completing this activity will be issued a certificate of participation. To participate in this journal CME activity: (1) review the learning objectives and author disclosures; (2) study the education content; (3) take the post-test with a $75 \%$ minimum passing score and complete the evaluation at http://www.medscape.org/journal/eid; and (4) view/print certificate. For CME questions, see page 393.

Release date: January 18, 2018; Expiration date: January 18, 2019

\section{Learning Objectives}

Upon completion of this activity, participants will be able to:

- Describe baseline characteristics and epidemiological features of patients with Pasteurella spp. Infection, according to a retrospective study and comprehensive review

- Determine characteristics of localized and invasive pasteurellosis, according to a retrospective study and comprehensive review

- Explain complications and mortality after localized and invasive pasteurellosis, according to a retrospective study and comprehensive review

\section{CME Editor}

Deborah Wenger, MBA, Copyeditor, Emerging Infectious Diseases. Disclosure: Deborah Wenger, MBA, has disclosed no relevant financial relationships.

\section{CME Author}

Laurie Barclay, MD, freelance writer and reviewer, Medscape, LLC. Disclosure: Laurie Barclay, MD, has disclosed the following relevant financial relationships: owns stock, stock options, or bonds from Pfizer.

\section{Authors}

Disclosures: Sándor Körmöndi, MD; Gabriella Terhes, MSc, PhD; Zoltán Pál, MD, PhD; Endre Varga, MD, PhD, DSc; Mária Harmati, MSc; Kriszina Buzás, MSc, PhD; and Edit Urbán, PharmD, PhD, Habil, DSc, have disclosed no relevant financial relationships.

Pasteurella spp. infection is becoming more noteworthy because of increasing numbers of pets in households and changes in pet-keeping habits. Our aim was to collect epidemiologic and clinical data about infections caused by Pasteurella spp. to achieve a comprehensive review. We studied 162 patients in Hungary who had positive Pasteurella

Author affiliations: University of Szeged, Szeged, Hungary

(S. Körmöndi, G. Terhes, Z. Pál, E. Varga, K. Buzás, E. Urbán);

Hungarian Academy of Sciences, Szeged (M. Harmati, K. Buzás)

DOI: https://doi.org/10.3201/eid2502.180641 spp. culture results during 2002-2015. An increasing tendency in rates of pasteurellosis could be observed from year to year. A total of $70(43.2 \%)$ cases were detected in patients $>60$ years of age. Localized infections caused by bites and scratches were the most common ( $n=114,70.4 \%)$; invasive infections were recorded in 48 patients $(29.6 \%)$. In localized infections, animal contact was common; in most invasive infections, the source of pasteurellosis could not be determined. The high number of complications and the unusually high death rate $(8 \%)$ emphasize the importance of diagnosing and treating human pasteurellosis. 
$P$ asteurella spp. infections can cause various diseases in wild and domestic animals; in humans, most infections are associated with cat or dog bites, licks, and scratches (1). Annually, in the United States, $\approx 300,000$ visits to emergency departments for animal bites or scratch wounds are recorded; however, not all of these are associated with infections. Regarding animal bites, 3\%-18\% of dog bites and $28 \%-80 \%$ of cat bites become infected $(2,3) ; 50 \%$ of dog bites and $75 \%$ of cat bites are associated with the presence of Pasteurella multocida (3), which can be frequently detected as part of the oral microbiota in various animals such as cats, dogs, pigs, and various wild animals $(2,3)$.

Data about injuries associated with animal bites or scratches in Hungary are not available; however, rabies vaccines are given to $>4,000$ patients annually (4,784 in 2017) (4). This number represents mainly cases in which the animal could not be observed after an accident or injury (4). Of the 35,000 traumatic injury cases (such as various injuries from accidents, bites, scratches, sport injuries, falls, and injuries that occur at home) that are recorded annually at our university hospital in Szeged, Hungary, 14-37 (0.04\%-0.1\%) are associated with animal bites or scratches.

Localized infections caused by Pasteurella spp. are characterized by cutaneous inflammation that usually develops shortly after animal bites or scratches. Later, local complications including osteomyelitis, septic arthritis, and abscess formation or systemic infection such as infection of large articulated joints, meningitis, intraabdominal infection, sepsis, and pneumonia may develop (5). In immunocompromised patients, the infection may manifest as severe pneumonia, sepsis, or a fatal form of pasteurellosis. In patients with underlying pulmonary disease, pneumonia, empyema, and lung abscess caused by Pasteurella spp. can be detected, whereas in patients with liver dysfunction, sepsis has been described (3). Other severe invasive infections (meningitis, endocarditis, and peritonitis) caused by various Pasteurella spp. have also been described; however, their incidences are rare $(6)$.

During the past 10 years, we have observed that the rate of human pasteurellosis is rising in Hungary, and in several cases of invasive pasteurellosis, animal contacts are usually not mentioned. In the literature, most papers dealing with human pasteurellosis are reports of individual cases; only a few papers have reported on all cases recognized in a hospital. To achieve better knowledge of human pasteurellosis, we collected data about both localized and invasive forms of human infections and compared the results with international data.

\section{Methods}

\section{Study Design and Patients}

We included patients in this study if they had microbiological investigation with positive results for Pasteurella spp. in the local university hospitals in Szeged, Hungary (intensive care unit and departments of traumatology, surgery, pediatrics, dermatology, ophthalmology, obstetrics and gynecology, otorhinolaryngology, and head and neck surgery), during 2002-2015. For culture, we used Columbia agar with 5\% sheep's blood and chocolate agar with PolyViteX (bioMérieux, https://www.biomerieux. com) and incubated the cultures at $37^{\circ} \mathrm{C}$ for $24 \mathrm{~h}$ in a $5 \% \mathrm{CO}_{2}$ incubator. We performed identification using the VITEK 2 GN ID and API NH Systems (bioMérieux) before 2012; after 2012, we used matrix-assisted laser desorption/ionization time-of-flight (MALDI-TOF) mass spectrometry (Bruker Daltonik, https:/www.bruker.com). We obtained patients' medical history from the local medical database. We identified local pasteurellosis if the patient had a superficial wound or tenosynovitis or if small joints were involved. Invasive pasteurellosis was present if large joints, including shoulder, hip, knee, or prostheses, were inflamed or if the patient had neurologic, pulmonary, cardiovascular, abdominal, or pelvic involvements or had septic shock or bacteremia.

This retrospective study was approved by the University Research Ethics Committee, Faculty of Medicine, University of Szeged, Hungary. Collection of data about participating patients was in accordance with ethical standards at the institutional and/or national research committee and with the 1964 Helsinki Declaration and its later amendments.

\section{Data Analysis}

We collected the following data from the medical database: age, sex, concurrent medical conditions, animal exposure history, outcomes, and laboratory findings at hospital admission. For statistical analysis, we collected and analyzed demographic and clinical data about the patients. We used descriptive statistics including means or medians with ranges and percentages to characterize data; in this case, we used Microsoft Excel 2013 (https:// www.microsoft.com). We used Mann-Whitney U-test or $\chi^{2}$ tests with Yates' correction to compare groups. A p value $<0.05$ was considered statistically significant. We analyzed the data using GraphPad Prism version 5.03 (https://www.graphpad.com/scientific-software/prism).

\section{Results}

\section{Baseline Characteristics of Patients with Pasteurella spp. Infection}

For 2002-2015, we isolated 211 Pasteurella spp. from 162 patients; we found that the number of isolates and human pasteurellosis cases has increased from year to year (Figure 1). The median age of patients with positive culture results was 57 (range 0-97) years. The proportion of Pasteurella spp. isolation was approximately equal for men $(n=78$, 


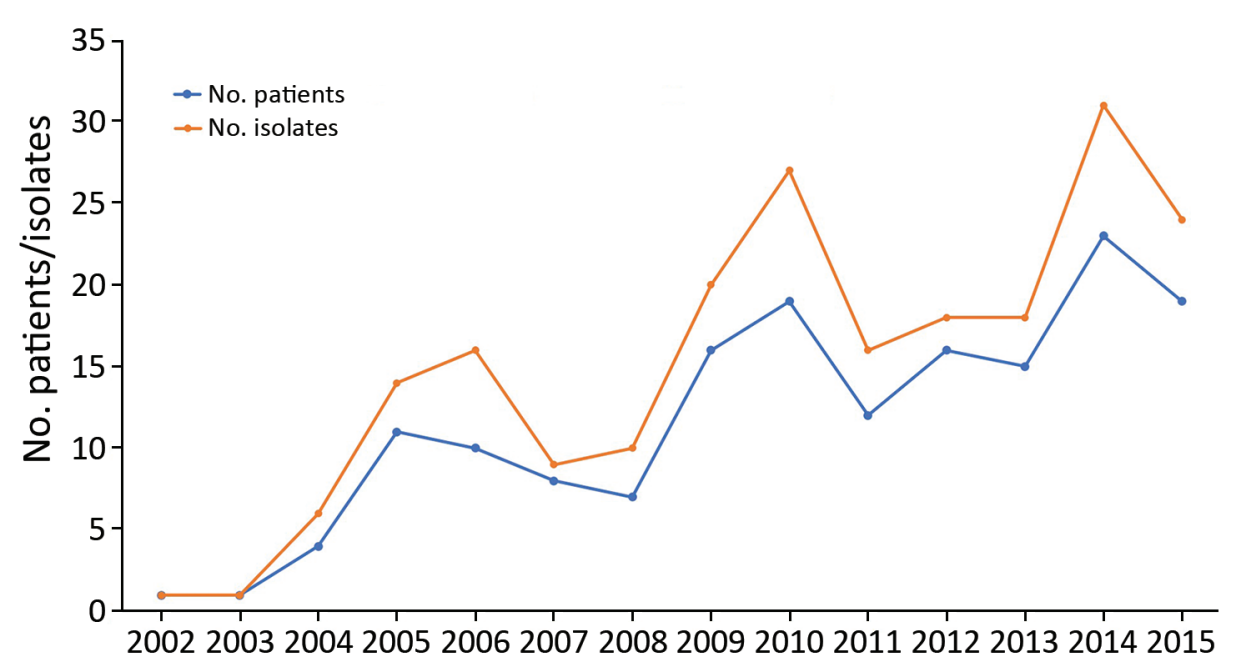

Figure 1. Increasing tendency in annual rates of human pasteurellosis and in the number of Pasteurella isolates, Szeged, Hungary, 2002-2015.

$48.1 \%)$ and women $(\mathrm{n}=84,51.9 \%)$, and we observed increasing rates of pasteurellosis cases by advanced age in both sexes (Figure 2).

We determined the distribution of Pasteurella spp. in clinical specimens (wounds, respiratory tract, and abdominal specimens; blood, synovial, cerebrospinal, and pleural fluids) (Table 1). Most strains $(\mathrm{n}=155,73.5 \%)$ were isolated from wound specimens (Table 2), but some strains originated from unusual sites such as synovial fluid, pleural fluids, and abdominal specimens. In the respiratory specimens $(n=23), 5$ strains were isolated from the upper respiratory tract; among these, asymptomatic carriages were considered in 3 patients. Two $P$. multocida strains isolated from the upper respiratory tract were also present in pleural fluid and bronchoalveolar lavage of the same patient with severe respiratory failure. In lower respiratory specimens, invasive infections were documented when pure cultures of Pasteurella spp. and high numbers of granulocytes were detected and the patient had symptoms of lower respiratory tract infections. All strains $(n=18)$ isolated from the lower respiratory specimens were grouped into the invasive infection cluster.

\section{Polymicrobial Pasteurella sp. Infections}

In 79 patients, Pasteurella sp. was the only isolate, whereas polymicrobial infections were detected in 83 patients $(51.2 \%)$. In these cases, the most frequent anaerobic bacteria isolated from clinical specimens were Fusobacterium nucleatum $(\mathrm{n}=18)$, Peptostreptococcus anaerobius $(\mathrm{n}=$ 11), Prevotella oralis $(\mathrm{n}=10)$, Prevotella melaninogenica $(\mathrm{n}=7)$, Prevotella loescheii $(\mathrm{n}=7)$, and Bacteroides pyogenes $(\mathrm{n}=7)$. Among facultative anaerobic bacteria, Staphylococcus aureus $(\mathrm{n}=12)$ and Escherichia coli $(\mathrm{n}=$ 5) were the most common species.

\section{Characteristics of Localized and Invasive Pasteurellosis}

Localized infections secondary to bites, scratches, or licking were the most prevalent type during the study period $(\mathrm{n}=114,70.4 \%)$. Most patients with localized infections were female $(n=68,59.6 \%)$. For invasive infections

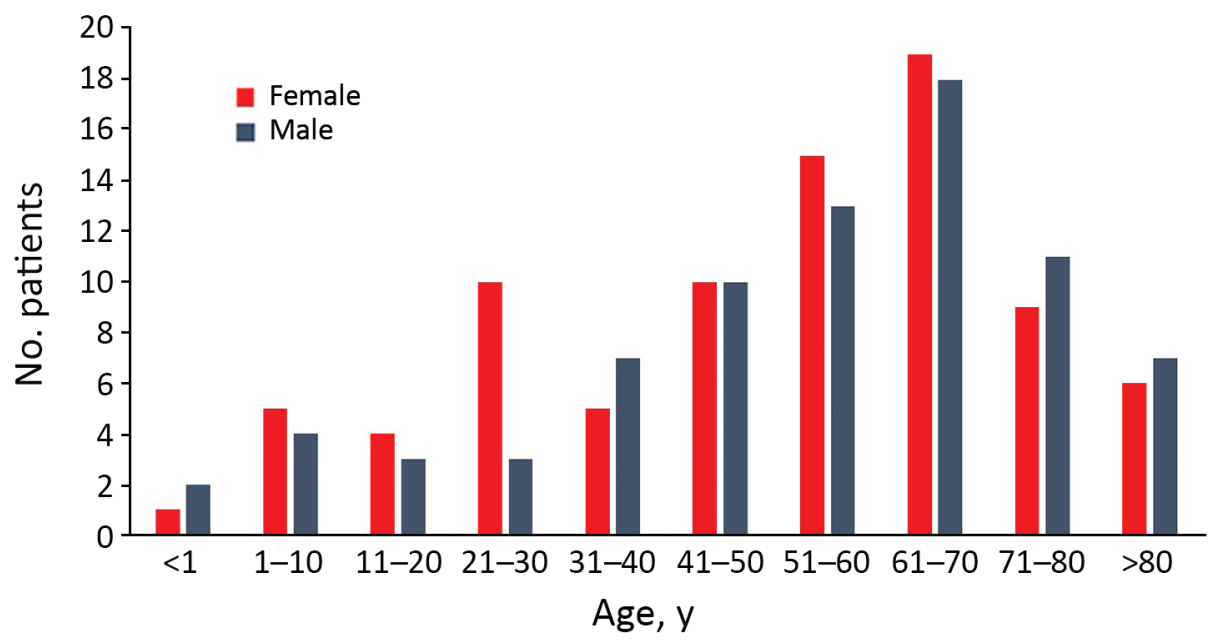

Figure 2. Distribution of pasteurellosis cases $(\mathrm{N}=162)$ according to age group and sex, Szeged, Hungary, 2002-2015. 
Table 1. Distribution of Pasteurella spp. in clinical specimens from patients in Szeged, Hungary, 2002-2015

\begin{tabular}{lc}
\hline Species & No. $(\%)$ isolated strains \\
\hline$P$. multocida & $160(75.8)$ \\
$P$. canis & $36(17.1)$ \\
$P$. pneumotropica & $11(5.2)$ \\
P. dagmatis & $2(0.9)$ \\
$P$. stomatis & $1(0.5)$ \\
$P$. aerogenes & $1(0.5)$ \\
\hline Total & $211(100)$ \\
\hline
\end{tabular}

$(\mathrm{n}=48)$, however, male predominance $(\mathrm{n}=32,66.7 \%)$ was observed, and that difference was statistically significant $\left(\chi^{2}=8.345, \mathrm{df}=1, \mathrm{p}=0.004\right)$. The difference in the age distribution among those with localized (median age 53.5 [range 0-97] years) and invasive (median age 63 [range 0 -87] years) infection was also statistically significant (Mann-Whitney $\mathrm{U}=2142 ; \mathrm{p}=0.029$ ). In the invasiveinfection group, the largest number of patients $(n=12$, $25 \%$ ) had various abscesses (e.g., liver abscess, pulmonary abscess, abscesses after sigmoid resection); 8 (16.7\%) patients had pneumonia, and $8(16.7 \%)$ had bacteremia. Respiratory failure was diagnosed in $6(12.5 \%)$ patients, osteomyelitis was in $4(8.3 \%)$ patients, pleurisy in 2 $(4.2 \%)$ patients, and arthritis in $2(4.2 \%)$ patients. Central nervous system infection, adnexitis associated with pelvic inflammatory disease, peritonitis, pacemaker infection, cirrhosis, and gangrene were also recorded.

In $8(7 \%)$ patients with localized infections and 37 (77.1\%) patients with invasive infections, underlying diseases were recorded in the medical history (Table 3); the difference between the 2 groups was statistically significant $\left(\chi^{2}=72.59, \mathrm{df}=1, \mathrm{p}<0.0001\right)$. Some patients with invasive infections had multiple underlying disorders. Cardiovascular disease, diabetes, and malignancy were the most frequent underlying diseases.

Of 114 patients with localized infection, 94 (82.5\%) had contact with a dog $(n=41,43.6 \%)$ or cat $(n=53$, $56.4 \%$ ). Ten patients had no animal contact, and no data were available for 10 patients. Animal contacts were recorded in $10(20.8 \%)$ of 48 cases of invasive infection; no information about animal contacts was available in 16 $(33.3 \%)$ cases. Twenty-two $(45.8 \%)$ of 48 patients with invasive pasteurellosis had no animal contact.

\begin{tabular}{lc}
\hline \multicolumn{2}{l}{ Table 2. Distribution of clinical specimens positive for Pasteurella } \\
spp. from patients in Szeged, Hungary, 2002-2015 \\
\hline Specimens & No. (\%) isolates \\
\hline Wound & $155(73.5)$ \\
Respiratory tract specimen & $23(10.9)$ \\
Blood & $14(6.6)$ \\
Synovial and pleural fluids & $4(1.9)$ \\
Abdominal & $3(1.4)$ \\
Cerebrospinal fluid & $1(0.5)$ \\
Others (middle ear, sinus, conjunctiva, & $11(5.2)$ \\
dialysis catheter) & \\
\hline Total & $211(100)$
\end{tabular}

Most injuries (74.6\%) in the localized-infection group were attributed to animal bites. Scratch wounds caused by cats were recorded in 12 patients; however, some of them had simultaneous bite injuries. Six cases of cat-associated injury were observed in women 21-30 years of age; in the same age group of male patients ( 3 patients), none had animal contact (Figure 2). Differences in the distribution of various Pasteurella spp. between localized and invasive infections could not be detected (Figure 3).

When we compared data about hospitalization in patients with localized and invasive infections, we found $71(62.3 \%)$ of 114 patients with localized infections had hospitalizations; the average length of stay was 8 (range 1-30) days. In the invasive-infection group, 40 of 48 patients $(83.3 \%)$ were hospitalized, and the average length of hospital stay was 12.7 (range 1-60) days. Patients with invasive infections were more frequently hospitalized than patients with localized infections $\left(\chi^{2}=5.999, \mathrm{df}=1, \mathrm{p}=\right.$ 0.014 ), and the average length of stay among patients with invasive pasteurellosis was also longer. Hospital admission was almost the same in patients with dog bites $(68.3 \%)$ and in patients with cat-induced injury (64.2\%). The average length of hospital stay was longer (11 [range 1-60] days) in cases of dog bites than in cases of cat bites or scratches $(8.3$ [range 3-41] days). In patients with localized infections, injuries affected mostly the upper extremities ( 75 patients, $65.8 \%$ ); in 23 patients, lower extremities were affected, mainly shins; in 6 patients, the face; in 1 patient, the eye; and in 1 patient, the genitals.

\section{Complications after Localized and Invasive Pasteurellosis}

Ileostomy, hysterectomy, skin transplantation, and amputation were documented in 4 cases of invasive pasteurellosis. Ileostomy was performed because of sudden onset of perforation; during the surgical procedure, a sample from the abdominal cavity was collected for microbiological culture, and P. multocida was isolated. Total abdominal hysterectomy and bilateral salpingo-oophorectomy were performed in a young woman with bilateral tubo-ovarian abscess due to P. multocida infection. A pacemaker electrode, a prosthesis, and a catheter were removed in 3 different cases because of endocarditis, recurrent inflammations around knee prosthesis, and purulent drainage around the dialysis catheter.

After localized Pasteurella sp. infection, severe complications were registered in 23 cases (20.2\%); functional disability, mainly in fingers, developed in 10 patients (6 cases associated with dog bites, 4 cases with cat bites and scratches); and lymphangitis was observed in 7 of 53 $(13.2 \%)$ patients. Following bites and scratches, 3 patients had amputations ( 2 from dog bites, 1 from a cat bite), affecting mainly the fingers; 3 patients had osteomyelitis (associated with dog bites) as a consequence of pasteurellosis. 
The high number of complications emphasizes the importance of localized and invasive pasteurella infections.

Of the 162 patients with pasteurellosis, $143(88.2 \%)$ patients recovered; in 6 cases, no data were available about the outcome of pasteurellosis (in these cases, patients were transferred to other hospitals). Thirteen of the 162 patients died; all these patients had invasive pasteurellosis infections. Seven of these 13 patients had pneumonia and respiratory failure, 5 patients had bacteremia, and 1 patient had panencephalitis after traumatic brain injury. The median age of patients who died was 69.5 (range 39-84) years; most of them had multiple underlying diseases. Laboratory investigations revealed elevated leukocytes, C-reactive protein levels, and liver enzymes in all patients who died from pasteurellosis. In 4 cases, animal contacts, including dogs, cats, and other animals, were recorded in the patient's medical history. No information about animal contact was available in 5 cases, and in 4 cases, no animal contact could be found. In cases of animal contacts, no bite or scratch was mentioned by the patients or relatives.

From clinical specimens (respiratory tract specimens, cerebrospinal fluid, and blood), P. multocida was isolated from 12 patients, and $P$. canis from 1 patient. All 13 of these patients were hospitalized; the average of length of hospital stay was 10.3 (range 1-30) days. Pasteurella sp. was isolated from respiratory specimens or pleural fluid from 6 patients, from blood culture from 4 patients, from 2 wound specimens, and from 1 cerebrospinal fluid specimen.

\section{Discussion}

According to data in the literature, two thirds of human infections are zoonotic in origin. Among these infections, human pasteurellosis is not a common cause of death in humans because of commonly used prophylactic treatment after animal bites or scratches; however, deaths caused by Pasteurella sp. infection have been increasing in the United States (3). We found that 162 patients had Pasteurella infections during 2002-2015 in our university hospital in Hungary. We found a higher number of cases caused by various Pasteurella species than similar surveys showed earlier, such as those by Giordano et al. (7) and Nollet et al. (5). To our knowledge, most publications dealing with human pasteurellosis showed only a few cases or reviewed only unusual infections caused by certain Pasteurella spp.; only 1 publication (5) presented data, from $\approx 102$ patients, to determine risk factors for invasive pasteurellosis. Those surveys showed that higher mean age and underlying diseases, mainly chronic liver disease and neoplasia, are commonly associated with invasive pasteurellosis. Our data confirmed that invasive infections are more frequent in elderly patients and that these patients usually have 1 or more underlying diseases.

Giordano et al. (7) and our results also showed that the detection of Pasteurella spp. from the blood or respiratory
Table 3. Presence of underlying disease in cases of local and invasive Pasteurella spp. infections among patients in Szeged, Hungary, 2002-2015

\begin{tabular}{lcc}
\hline & \multicolumn{2}{c}{ No. (\%) patients } \\
\cline { 2 - 3 } Underlying disease & Local infection & Invasive infection \\
\hline Malignancy & 0 & $10(20.8)$ \\
Cardiac & 0 & $6(12.5)$ \\
Diabetes mellitus & $4(3.5)$ & $6(12.5)$ \\
Pulmonary & 0 & $2(4.2)$ \\
Hepatic (cirrhosis) & 0 & $2(4.2)$ \\
Prosthesis & 0 & $2(4.2)$ \\
Psychiatric & $1(0.9)$ & $1(2.1)$ \\
Genetic (Down & 0 & $1(2.1)$ \\
syndrome) & & \\
Multiple & $1(0.9)$ & $7(14.6)$ \\
No data & $106(93)$ & $11(22.9)$ \\
\hline Total & $114(100)$ & $48(100)$ \\
\hline
\end{tabular}

tract was frequently associated with the absence of animal bites; at the same time, skin infections were usually attributed to animal bites. Most hospitalized patients with animal bites were admitted because of cat bites. Patients without animal bites were more frequently hospitalized, and their length of stay was longer, compared with patients who had animal bites. Patients treated in the ICU did not have animal bites, and most of them had no animal contact, as well. In our study, among patients with invasive pasteurellosis, the death rate was $27.1 \%$. Similarly, the death rate was $21 \%$ in Giordano et al.'s observational study (7); in Nollet's study, the death rate from invasive infections was $11 \%$ and the death rate for localized infections was $1.4 \%(5)$.

The French Pasteurella National Center has reported that the prevalence of central nervous system infection caused by Pasteurella spp. is $<1 \%$ of all Pasteurella infections (8). In adults, meningitis caused by Pasteurella infection is usually associated with cranial trauma or surgery or chronic otitis (9). Our data confirmed these findings. The prevalence of central nervous system infection caused by Pasteurella sp. was $0.6 \%$. One patient with meningitis had a traumatic injury to his head from a car accident and was found near a stable; thus, the source of this infection was deemed to be the environment.

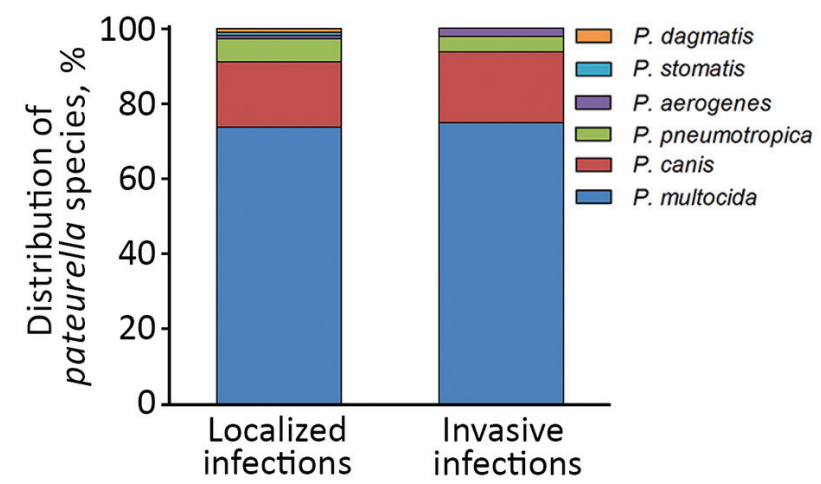

Figure 3. Distribution of various Pasteurella spp. in localized and invasive infections, Szeged, Hungary, 2002-2015. 
In the literature, dog bite is the most common cause of bite injury, followed by cat bite. Most bite injuries are minor wounds; thus, patients generally do not seek medical attention. Therefore, the reported prevalence of biterelated wounds is probably the tip of the iceberg. Dog bites may be associated with fractures because of their high energy, whereas cat bites frequently cause puncture wounds, leading to the development of tenosynovitis (10). Wounds caused by dog bites are less frequently infected than wounds caused by cat bites. Our investigations supported this observation, because patients with cat bites or scratches have pasteurellosis more frequently than do patients with dog bites. Literature data show that cat bites or scratches are more dangerous than dog bites because the injuries tend to be deep and are therefore difficult to clean properly (2). When we studied the complications following dog or cat bites, we observed that after dog bites, the rates of functional disability, osteomyelitis, and amputation were slightly higher than for those after cat bites. Lymphangitis could not be detected after dog bites; this finding was not described earlier.

P. multocida subsp. multocida is the most frequent clinical Pasteurella isolate in humans, ahead of $P$. canis and P. multocida subsp. septica (11). This fact was also confirmed by our investigations; $68.7 \%$ of Pasteurella strains were $P$. multocida, the second most common species was $P$. canis $(17.1 \%)$, and third was $P$. pneumotropica (5.2\%). Differences between species isolated from localized and invasive infections could be detected in our study, a finding also confirmed by Nollet et al. (5).

We followed the rates of human pasteurellosis and changes in the number of Pasteurella isolates from year to year, and we observed increasing tendencies in both groups. This finding may be explained by the increasing number of animals in households, and close intimate contact with pets. Approximately $57 \%$ of households in Canada own $\geq 1$ companion animal, whereas only $39 \%$ of persons $>65$ years of age have a pet (12). Similar data are not available in Hungary, but according to unofficial data, $>3$ million dogs and $\approx 3$ million cats live in Hungary (human population 9.938 million). In the Canada survey, the rate of pet ownership was the highest among middle age person (12), and pet-associated infections usually originated from injuries or animal bites; we saw a similar trend in our survey. In elderly patients, because of age-related dysfunction of the immune system or underlying disease, the risks for pet-associated disease and invasive infections are increased.

Another possible explanation for the increasing tendency in the rate of human pasteurellosis is the development of microbiological methods for identification, such as use of MALDI-TOF mass spectrometry and molecular methods. However, the possible role of these methods in explaining the higher number of isolates or human pasteurellosis cases could be excluded in study because we started to use MALDI-TOF mass spectrometry after 2012 but the increasing tendency in pasteurellosis could be observed before 2012. In addition, the spectrum of isolated various Pasteurella spp. did not change after the introduction of MALDI-TOF mass spectrometry and molecular methods; earlier automated identification systems provided adequate identification of Pasteurella strains.

The retrospective nature of this study and data interpretation have some limitations. Medical records for inpatients are completed better than those for outpatients; this may lead to an underestimate of concurrent conditions for outpatients, who fall mostly into the localized-infection group. In several cases, medical records did not contain information about animal contact (mainly in case of nonbite-associated pasteurellosis), which may also cause underestimation of the number of animal contacts. The number of infections caused by Pasteurella spp. is probably higher than we observed, because patients, mainly those in small villages, can receive emergency treatment from a local general practice physician.

Pets, including dogs and cats, are frequently recommended to patients with chronic illness because animal therapy might provide a potential health benefit. Elderly patients may also own companion animals to combat loneliness; however, pets may be the potential source of various infections or injuries (13). Our retrospective survey showed that the rate of human pasteurellosis over a 13-year study period in Hungary increased from year to year and with advanced age, and the number of Pasteurella infections was higher than it was in earlier studies. We observed that Pasteurella patients with cat- and dogassociated injuries were frequently hospitalized. In cases of invasive infections, the source of Pasteurella infection was frequently unknown. In spite of the adequate treatment on the basis of medical chart review and antimicrobial drug susceptibility of the isolated strain, the death rate from these infections was $27.1 \%$ in our study. We also found that complications after localized infections were detected frequently, and certain complications, such as lymphangitis, are associated only with injuries caused by cat bites; this connection was not described in earlier publications. We think that the rate of pasteurellosis is much higher than estimated because many patients with smaller injuries do not seek medical advice, and in many cases, general practice physicians try to treat smaller injuries and do not perform sample collection or order microbiological investigations. Our results, as well as international results, show that education about the possible health hazards associated with pet ownership should be provided, and the increased risks for infection in elderly and immunocompromised patients should be emphasized. 


\section{About the Author}

Dr. Körmöndi is an orthopedic surgeon in the Department of Traumatology, University of Szeged, Szeged, Hungary. His primary research interests are studies on injuries commonly affecting the musculoskeletal system, use of cutting-edge methods and treatment techniques in traumatology, investigation of bacterial infections following traumatic injury, and treatment of surgical site infections.

\section{References}

1. Shirzad Aski H, Tabatabaei M. Occurrence of virulence-associated genes in Pasteurella multocida isolates obtained from different hosts. Microb Pathog. 2016;96:52-7. http://dx.doi.org/10.1016/ j.micpath.2016.04.008

2. Christenson ES, Ahmed HM, Durand CM. Pasteurella multocida infection in solid organ transplantation. Lancet Infect Dis. 2015;15:235-40. http://dx.doi.org/10.1016/S1473-3099 (14)70895-3

3. Wilson BA, Ho M. Pasteurella multocida: from zoonosis to cellular microbiology. Clin Microbiol Rev. 2013;26:631-55. http://dx.doi.org/10.1128/CMR.00024-13

4. Megbetegedési veszély esetén kötelező védőoltások Magyarországon a 2017. évben. EMMI Kórházhigiénés és Járványügyi Felügyeleti Főosztály, editor. Védőoltás 2017. OSAP 1566. 2018 [cited 2018 Sep 3]. https://www.antsz.hu/data/ cms87737/Vedooltas 2017.pdf

5. Nollet V, Souply L, Rosolen B, Mohseni-Zadeh M, Martinot M. Risk factors for invasive pasteurellosis: a retrospective case study. Eur J Clin Microbiol Infec Dis. 2016;35:1975-81 https://dx.doi.org/10.1007/s10096-016-2749-y

6. Branch J, Kakutani T, Kuroda S, Shiba Y, Kitagawa I. Pasteurella multocida infective endocarditis: a possible link with primary upper respiratory tract infection. Intern Med. 2015;54:3225-31. http://dx.doi.org/10.2169/internalmedicine.54.4973

7. Giordano A, Dincman T, Clyburn BE, Steed LL, Rockey DC Clinical features and outcomes of Pasteurella multocida infection. Medicine (Baltimore). 2015;94:e1285. http://dx.doi.org/10.1097/ MD.0000000000001285

8. Escande F, Lion C. Epidemiology of human infections by Pasteurella and related groups in France. Zentralbl Bakteriol. 1993;279:131-9. http://dx.doi.org/10.1016/S0934-8840(11)80499-8

9. Kumar A, Devlin HR, Vellend H. Pasteurella multocida meningitis in an adult: case report and review. Rev Infect Dis. 1990;12:440-8. http://dx.doi.org/10.1093/clinids/12.3.440

10. Raval P, Khan W, Haddad B, Mahapatra AN. Bite injuries to the hand-review of the literature. Open Orthop J. 2014;8:204-8. http://dx.doi.org/10.2174/1874325001408010204

11. Holst E, Rollof J, Larsson L, Nielsen JP. Characterization and distribution of Pasteurella species recovered from infected humans. J Clin Microbiol. 1992;30:2984-7.

12. Alberta Ministry of Agriculture and Rural Development. Consumer corner: Canadian pet market outlook, June 2014 [cited 2018 Jan 8]. http://www1.agric.gov.ab.ca/\$department/deptdocs.nsf/all/ sis14914/\$file/sarah_pet_june20_2014.pdf

13. Cherniack EP, Cherniack AR. The benefit of pets and animalassisted therapy to the health of older individuals. Curr Geronotol Geriatr Res. 2014;2014:623203. http://dx.doi.org/10.1155/ $2014 / 623203$

Address for correspondence: Gabriella Terhes, University of Szeged, Institute of Clinical Microbiology, Semmelweis Street 6, Szeged H-6725 Hungary; email: terhesga@gmail.com

\section{EID Podcast: A Worm's Eye View}

Ben Taylor, cover artist for the August 2018 issue of EID, discusses how his personal experience with the Loa loa parasite influenced this painting.
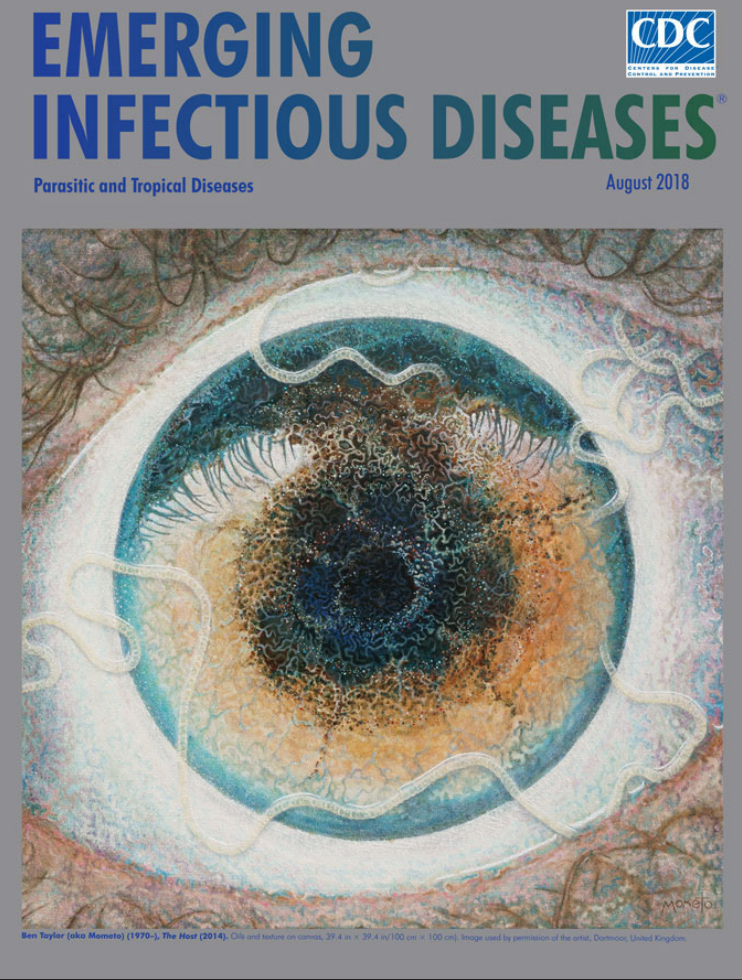

Visit our website to listen: https://tools.cdc.gov/medialibrary/ index.aspx\#/media/id/392605

EMERGNG INFECTIOUS DISEASES 